\title{
INFLUENCE OF RADIATION ON THE DIELECTRIC PROPERTIES OF XLPE BASED INSULATION SYSTEMS
}

\author{
Vladimír Ďurman — Jaroslav Lelák *
}

\begin{abstract}
The paper discusses the possibilities of acquiring and processing the dielectric data in the range of very low frequency (VLF) and also design and construction of an impedance analyzer for measurements in this frequency range. The impedance measurements in the VLF range were used for investigation of the influence of radiation on the cross-linked polyethylene cable dielectric. An expressive $\beta$-type relaxation process was found in this type of cable. Most of the dielectric response parameters of the process depend significantly on the absorbed dose of radiation. The analysis of the process parameters showed that the most probable reason of the structural changes in cross-linked polyethylene was additional cross-linking. The results also proved that the impedance measurements in the VLF range could be effectively used in practice for estimation of the absorbed dose in the cross-linked polyethylene cables stressed by radiation.t data only. In order to identify or classify motion, data processing in real time is needed.
\end{abstract}

K e y w or d s: cable diagnostics, dielectric spectroscopy, very low frequency, relaxation

\section{INTRODUCTION}

Diagnostic methods based on dielectric measurements in the time and frequency domains are preferably used for investigation of the degradation degree in power cables during their operation. In the frequency domain, dissipation factor $(\tan \delta)$ measurement is frequently used as a diagnostic tool. In the time domain the absorption current or recovery voltage can be measured. From these quantities some derived parameters such as the polarization index are calculated for routine cable evaluation. The insulation resistance calculated from the steady-state value of the absorption current also belongs to the very important cable characteristics. The parameters acquired by the diagnostic methods mentioned above can individually reflect the changes caused by the long-term operation or the changes induced by artificial ageing. In this paper the measurements of the impedance are used for detecting the cross-linked polyethylene (XLPE) cable degradation caused by radiation. Cables insulated with cross-linked polyethylene are used widely for transmission and distribution purposes and also for other special applications. Because of its low permittivity and $\tan \delta$, XLPE is considered as an excellent insulating material. Like many other materials, it experiences structural degradation in humid environment. This type of degradation has already been observed and quantified. Well-known are the ageing processes under the electric and the thermal stress. But there is not much experience with XLPE behaviour in a gamma-irradiated stage. Radiation can worsen but also enhance the electrical properties of the XLPE dielectric. Research in this field is necessary for the future use of XLPE cables in nuclear power stations.

\section{DIELECTRIC POLARIZATION}

The dynamic properties of a linear system are usually represented by three characteristics:

a) The impulse response $h(t)$ - response of the system resulting from the unit impulse $\delta(t)$.

b) The step response $g(t)$ - response of the system resulting from the unit-step function $u(t)$.

c) The transfer function $H(p), H(\omega)$ - the ratio of the Laplace (or the Fourier) transform of the output signal and the transform of the input signal.

In the theory of dielectrics the electric field $E(t)$ is considered as the system input and the polarization $P(t)$ as the system output [1]. The system response to the step of electric field $E_{0} u(t)$ is

$$
P(t)=E_{0} \int_{0}^{t} h(\lambda) d \lambda .
$$

In experiments the polarization $P(t)$ is not measured directly. A commonly used technique in this field is the measurement of the so-called relaxation (absorption) current after applying a step voltage. The current density can be evaluated from Maxwell equations. Let $\gamma_{0}$ denote the conductivity of the dielectric. After application of a step electric field $E_{0} u(t)$ at a time $t=0$ the current density $J(t)$ is given by

$$
\begin{aligned}
& J(t)=\gamma_{0} E_{0}+\varepsilon_{0} \frac{d E(t)}{d t}+E_{0} \frac{d}{d t} \int_{0}^{t} h(\lambda) d \lambda \\
& =\gamma_{0} E_{0}+\varepsilon_{0} E_{0} \delta(t)+E_{0} h(t) .
\end{aligned}
$$

\footnotetext{
* Slovak University of Technology in Bratislava, Faculty of Electrical Engineering and Information Technology, Department of Electrotechnology, Ilkovičova 3, 81219 Bratislava; vladimir.durman@stuba.sk, jaroslav.lelak@stuba.sk
} 
Dividing (2) by $E_{0}$ we get a formal expression for the time dependence of conductivity after application of a step electric field

$$
\gamma(t)=\gamma_{0}+\varepsilon_{0} \delta(t)+\gamma_{a}(t)
$$

The conductivity step response is given by the sum of the steady-state (DC) conductivity, the unit impulse and the absorption conductivity $\left(\gamma_{a}\right)$ characterizing the dielectric relaxation process. The absorption conductivity is part of the current step response and it equals to the polarization impulse response

$$
\gamma_{a}(t)=h(t) .
$$

When changing from the time domain to the frequency domain, the relationship between the transfer function and the impulse response is preferably used. As the Fourier transform of the unit impulse is 1 , the transfer function can be computed directly as the Fourier transform of the impulse response

$$
\frac{\tilde{P}(\omega)}{\tilde{E}(\omega)}=\int_{0}^{\infty} h(t) e^{-j \omega t} \mathrm{~d} t
$$

where the symbols with a tilde denote complex variables and $\mathrm{j}$ is the complex unit.

The term on the left side of (5) is the product of the complex susceptibility and the free-space permittivity $\tilde{\chi} \varepsilon_{0}$. Using the relationship between the complex susceptibility and the relative complex permittivity $\left(\tilde{\varepsilon}_{r}=1+\tilde{\chi}\right)$, we have

$$
\tilde{\varepsilon}(\omega)=\varepsilon^{\prime}-j \varepsilon^{\prime \prime}=\varepsilon_{\infty}+\int_{0}^{\infty} \gamma_{a}(t) e^{-j \omega t} \mathrm{~d} t .
$$

where $\varepsilon_{\infty}$ is the contribution of the fast polarizations to the overall permittivity.

An important step in dielectric data evaluation is a proper choice of the dielectric response approximation. The empirical responses of Cole-Cole or Havriliak-Negami $[2,3]$ are suitable in many cases. Recently, the universal response of Jonscher is preferred [4]. Unfortunately, this response cannot be expressed in an analytical form. Instead of it, an approximation can be used as [5]

$$
h(t)=\Delta \varepsilon\left[e^{-\frac{t}{\tau}}\left(\frac{t}{\tau}\right)^{-m}+\left(1-e^{-\frac{t}{\tau}}\right)\left(\frac{t}{\tau}\right)^{-n}\right],
$$

where $\Delta \varepsilon=\left.\varepsilon(\omega)\right|_{0}-\left.\varepsilon(\omega)\right|_{\infty}$ is the permittivity increment of the investigated process, $\tau, m$ and $n$ are the dielectric response parameters. Parameters $m$ and $n$ are bounded to intervals: $0<m<1,0<n<2$. After using the Fourier transform of response (7) we have

$$
\begin{aligned}
\tilde{\varepsilon}(\omega)= & \varepsilon_{\infty}+\Delta \varepsilon\left[\frac{\tau^{m} \Gamma(1-m)}{\left(\frac{1}{\tau}+j \omega\right)^{1-m}}-\right. \\
& \left.\frac{\tau^{n} \Gamma(1-n)}{\left(\frac{1}{\tau}+j \omega\right)^{1-n}}+\frac{\tau^{n} \Gamma(1-n)}{(j \omega)^{1-n}}\right],
\end{aligned}
$$

where $\Gamma$ is the Gamma function.

We have to note that also the Havriliak-Negami function, which is expressed as

$$
\tilde{\varepsilon}(\omega)=\varepsilon_{\infty}+\frac{\Delta \varepsilon}{\left(1+(j \omega \tau)^{1-\alpha}\right)^{\beta}}
$$

has some properties of the universal Jonscher response. Namely, the frequency dependence of the permittivity loss component is linear in a log-log scale outside the frequency region where a maximum appears. For the shape parameters, and $\mathrm{m}, \mathrm{n}$ of the above functions the next equalities can be used $n=(1-\alpha) \beta+1, m=\alpha$.

The five parameters in (9) $-\varepsilon_{\infty}, \Delta \varepsilon, \tau, \beta, \alpha$ depend on temperature. A good approximation for the temperature dependence of all parameters except $\tau$ is the next relation

$$
p(T)=p_{\mathrm{REF}}\left(1+k_{p}\left(\frac{1}{T}-\frac{1}{T_{\mathrm{REF}}}\right)\right),
$$

where $T$ is temperature, $p$ is one of the above parameters, $p_{\mathrm{REF}}$ is the value of parameter $p$ at temperature $T_{\mathrm{REF}}$ and $k_{p}$ is the temperature coefficient [6].

The temperature dependence of parameter $\tau$ acquires different shapes for various relaxation mechanisms [7]. Mostly it follows the Arrhenius law

$$
\tau(T)=\tau_{\infty} \exp \left(\frac{W_{A}}{R T}\right)
$$

where $W_{A}$ is the activation energy of the relaxation process, $R$ is the gas constant and $\tau_{\infty}$ is the limit of $\tau$ for $T \rightarrow \infty$. The temperature dependence of $\tau$ for relaxation processes in many polymers obeys the VogelFulcher-Tammann (VFT) law

$$
\tau(T)=\tau_{\infty} \exp \left(\frac{W_{V}}{R\left(T-T_{V}\right)}\right)
$$

where $T_{V}$ is the so-called ideal glass transition or Vogel temperature. In fact, $T_{V}$ values are about $30-70 \mathrm{~K}$ below the glass transition temperature of polymers.

\section{DIELECTRIC PROPERTIES OF POLYMERS}

Polymers are materials of a complex structure which comprises long chains consisting of dipoles with different size and orientation. Each group of dipoles contributes to the overall relaxation process by a separate part that is visible as a peak in the frequency dependence of the loss factor. Individual relaxation processes were formerly identified by symbols $\alpha, \beta, \gamma$ depending on the peak position in the frequency or temperature scale. The $\alpha$-process matches to the peak at the 
lowest frequency (for the constant-temperature measurements) or to the peak at the highest temperature (for the constant-frequency measurements). This is just a simple classification which requires some explanation concerning the polymer structure [7]. The groups of dipoles can be classified according to their placement in the polymer backbone and also according to the type of their motion in the electric field. There are two possibilities of dipole placement toward the backbone: parallel and perpendicular. The dipoles which are not components of the backbone (eg dipoles in the side groups) are arbitrarily oriented. As for the dipole motion, three possibilities can appear: localized motion (at the atom level), segmental motion (at the level of a macromolecule part) and chain motion (motion of the whole molecule).

With the help of the above classification it was found that the $\alpha$-process is based on the segmental motion. This relaxation process is a cooperative phenomenon, $i e$ the motion of a selected segment influences the neighbour part of the macromolecule and the neighbourhood in feedback affects the original segment. The $\alpha$-process is caused mostly by the dipoles with perpendicular orientation toward the backbone [7]. The temperature dependence of its relaxation time does not obey the Arrhenius law but the Vogel-Fulcher-Tammann (VFT) equation [6]. In addition to the ordinary $\alpha$-process, a similar type of relaxation exists in polymers comprising the dipoles with parallel orientation toward the backbone. It is called the normal mode relaxation and it is based on the chain motion. The relaxation frequency of this process appears below the frequency of the $\alpha$-process.

The second important relaxation process in polymers is the $\beta$-process. Commonly, it is connected with the segmental motion of the dipoles in the side groups. The relaxation frequency of this process is higher as compared with the $\alpha$-process. The relaxation time obeys the Arrhenius law. The permittivity increment $\Delta \varepsilon$ in the complex permittivity functions $(8,9)$ is less for the $\beta$-process than the one for the $\alpha$-process and the temperature coefficient of this increment (equation 10) is negative for the $\beta$-process and positive for the $\alpha$-process. Explanation of this behaviour can be found in disequilibria between two temperature influenced processes: reduction of the dipole concentration with temperature at one side and an increase of the movable dipoles due to release of the intermolecular bonds on the other side. The first process prevails in the case of $\beta$-processes and the second one in the case of $\alpha$-processes [7].

In relation with the structure of the side groups in polymers, more than one $\beta$-process can be recognized in the relaxation spectrum. These processes are then denoted as $\gamma$ or $\delta$. We can distinguish these processes by their activation energy. The approximate values of energies are 85,20 and $5 \mathrm{~kJ} \mathrm{~mol}^{-1}$ for the $\beta-\gamma$ - and $\delta$ processes respectively.

\section{EXPERIMENT}

\subsection{Construction of the impedance analyzer}

The impedance measurement methods were in the past based predominantly on the bridge principle. The bridge balancing is not a very fast and easy procedure especially at a very low frequency. Automation of the balancing process is also questionable. That is why the bridges were replaced by equipment for straight current and voltage measurement.

Recent equipment has a source of harmonic voltage which is applied to the measured object along with scanning the current by a current-voltage converter. The absolute values of the voltage on a specimen and the voltage transposed from the current are converted to digital signals. The impedance is calculated from the absolute values and the phases of voltages in the equipment control unit.

In the range of very low frequencies (VLF) the impedance can be measured also by the step-voltage response of the object. The response in the form of the absorption current can be converted into the frequency domain by using the Fourier transform (equation 6). This type of calculation is sometimes not very precise as the voltage is of exponential or oscillatory form instead of the step form. In objects with a high geometric capacitance an additional error can appear due to the charging current flowing through the equipment input resistance. The input resistance is also source of errors in the case of switching the instrument ranges during absorption current measurement.

The method using the harmonic voltage is relative slow comparing with the absorption current method. The voltage must be applied to the specimen more than just for one period to ensure elimination of undesirable transient phenomena. The object impedance is then calculated by the harmonic analysis. This seeming disadvantage has in turn also a positive impact. By finding the higher harmonics we can check the object linearity.

Our laboratory impedance analyzer is based on utilization of some standard electronic components and equipment (Fig. 1).

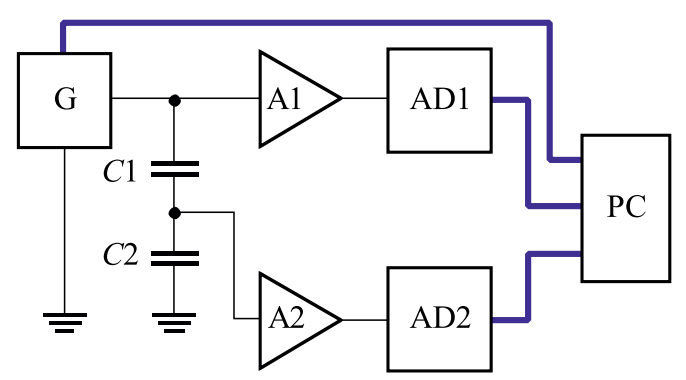

Fig. 1. The impedance analyzer block diagram (explanation of the symbols is in the text below) 


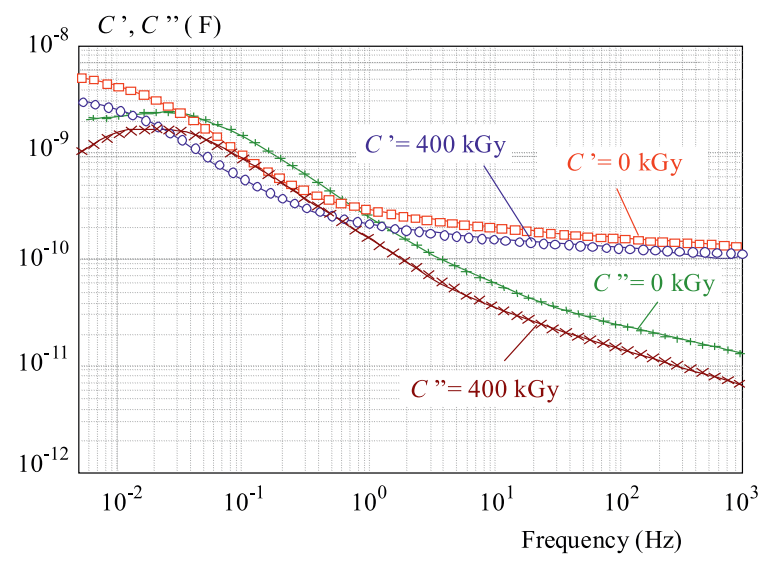

Fig. 2. Frequency dependence of the complex capacitance components at $323 \mathrm{~K}$

We use the RIGOL DG2021A Function/Arbitrary waveform generator as a source of harmonic voltage (it is assigned as G in Fig. 1). The voltage is connected to the divider consisting of the capacitance normal $(\mathrm{C} 1)$ and the measured object $(\mathrm{C} 2)$. Both analog voltages are scanned by ultra-low bias current operational amplifiers OPA 129 (A1, A2). After scanning, the voltages are converted to the digital form in the 16-bit digital units (AD1, AD2). The whole equipment is controlled by a computer (PC) through the GPIB and AD controller. The analyzer was checked by measuring the impedances of known values. The upper limit of the measuring frequency in this set is given by the throughput of the operational amplifiers and digital converters. Its value is about $1 \mathrm{kHz}$. The lower frequency limit is given only by the waveform generator and the long-term stability of all components. In practice, the value of $0.001 \mathrm{~Hz}$ can be reached without difficulty.

The important parameter that must be taken into account especially in the range of VLF is the number of periods intended for one measurement. The number of periods can be estimated by modelling the transient arising after the voltage application. For modelling we can use equation (2) modified for an arbitrary voltage as

$$
\begin{array}{r}
i(t)=\frac{C_{G}}{\varepsilon_{0}}\left[\gamma_{0} U(t)+\varepsilon_{0} \varepsilon_{\infty} \frac{\mathrm{d} U(t)}{\mathrm{d} t}+\right. \\
\left.+\frac{\mathrm{d}}{\mathrm{d} t} \int_{0}^{t} h(t-\lambda) U(\lambda) \mathrm{d} \lambda\right],
\end{array}
$$

where $i(t)$ is the current flowing through the specimen during measurement, $C_{G}$ is the geometric capacitance and $U(t)$ is the input voltage. If $U(t)$ is a harmonic voltage and the dielectric response has the frequently used formula according to Havriliak-Negami, then the Laplace transform of equation (13) can be solved by means of the numeric inverse Laplace transform [8]. The results of modelling show that the current has a harmonic course beginning from the third period of the applied voltage. This is valid in the whole range of frequencies mentioned

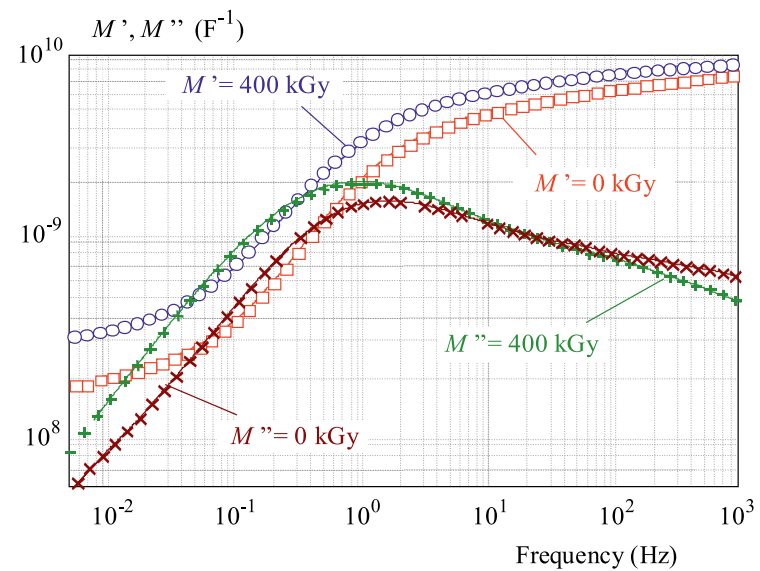

Fig. 3. Frequency dependence of the complex module components at $323 \mathrm{~K}$

above. These results were proved by analysis of the currents in the real and the model impedances. A minimum precision of $2 \%$ was achieved at comparative measurements of the real and imaginary part of impedance.

\subsection{Specification of specimens and the data processing}

Specimens of the length $100 \mathrm{~cm}$ were cut from a fourcore XLPE cable and irradiated to get define dose of radiation. Four different doses were chosen (100, 200, 300 and $400 \mathrm{kGy}$ ). The source of radiation was a gamma-emitter ${ }^{60} \mathrm{Co}$ with the dose rate of $950 \mathrm{~Gy} \mathrm{~h}^{-1}$. The irradiated specimens were compared with a non-irradiated specimen from the same cable.

The three cores of each specimen were short connected to create one electrode of the system. The rest core created the second electrode. An impedance of this electrode system was measured in the frequency range $5 \mathrm{mHz}-1$ $\mathrm{kHz}$ at temperatures from $30{ }^{\circ} \mathrm{C}$ to $90^{\circ} \mathrm{C}$ by means of the impedance analyzer described above. The voltage on the specimens during these measurements was $2 \mathrm{~V}$.

To process the impedance data in the way familiar in the dielectric data analysis we used a parameter known as a complex capacitance $\tilde{C}(\omega)$

$$
\tilde{C}(\omega)=\frac{1}{j \omega \tilde{Z}}=\frac{C_{G} \varepsilon^{\prime}-j C_{G} \varepsilon^{\prime \prime}}{\varepsilon_{0}}=C^{\prime}-j C^{\prime \prime},
$$

where $\tilde{Z}$ is the impedance, $C_{G}$ is the geometric capacitance of the specimen and $C^{\prime}, C^{\prime \prime}$ are the real and the imaginary part of the complex capacitance $\tilde{C}(\omega)$. The measured impedances for each specimen were transformed to the complex capacitances and the unknown parameters in (8) or (9) were optimized using the NelderMead type simplex search method. The optimization criterion was the sum of squares of differences between the measured and calculated values of $C_{G} \varepsilon^{\prime} / \varepsilon_{0}$ along with the differences of $\tan (\delta)$. Next, the temperature dependence of the optimized parameters for each specimen was fitted by equations (10) and (11) with help of the linear regression method. 


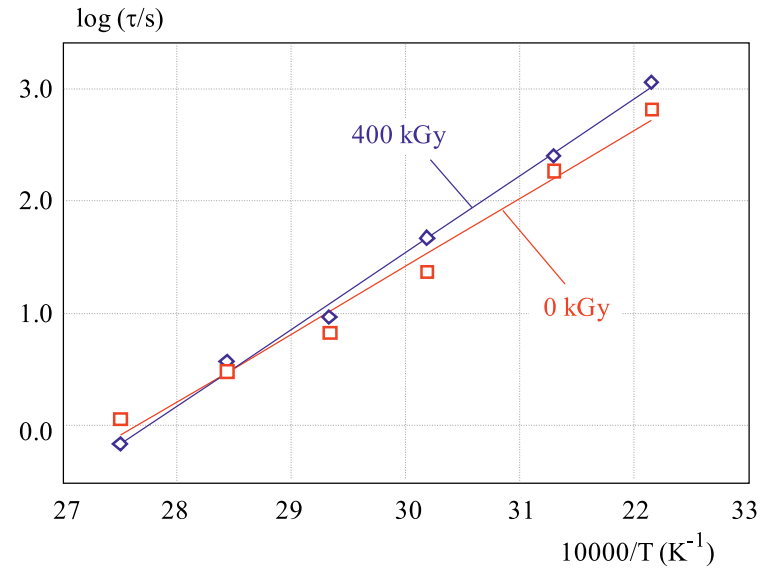

Fig. 4. The Arrhenius plot of the relaxation time for selected specimens

After finishing the data processing we found that the fitting function (9) yielded better results concerning the overall sum of squares comparing with the function (8). That is why we present in the next section only the results for Havriliak-Negami function.

\section{RESULTS AND DISCUSSION}

The calculated data of the complex capacitance are in Fig. 2. For the sake of transparency we show just the curves for the minimum and maximum absorbed dose.

The low frequency region of the observed process can be better resolved by displaying the data in the form of the complex modulus $\tilde{M}=M^{\prime}+j M^{\prime \prime}$, which is simply the reciprocal value of the complex capacitance (Fig. 3).

A relaxation process is clearly seen in both of these figures with a peak in the loss component of capacitance at about $3 \times 10^{-2} \mathrm{~Hz}$. The peak is smaller for the irradiated specimen, but its shift in the frequency scale is negligible. The frequency dependence of the loss components in Figs. 2 and 3 is not linear in the logarithmic scale at higher frequencies, in contrary to equations (8) and (9). The deflection from linearity is known in literature as an "excess wing" or "high frequency wing" [9]. There is no unambiguous explanation of the excess wing background.
The most probable interpretation of the wing is a presence of another relaxation process with very small permittivity increment. This process is almost completely overlapped by the relaxation process in its neighbourhood. The main relaxation process in our case was analyzed by the temperature dependence of its parameters. Temperature dependence of the relaxation time follows the Arrhenius law. It is depicted for selected specimens in Fig. 4. The corresponding values of $\tau_{\infty}$ and $W_{A}$ for all specimens are in Table 1. Temperature dependences of other parameters from equation (9) approximated by equation (10) are in Table 2.

Table 1. Variation of Arrhenius parameters with absorbed dose

\begin{tabular}{lcc}
\hline $\begin{array}{l}\text { Absorbed } \\
\text { dose } \\
(\mathrm{kGy})\end{array}$ & $\begin{array}{c}\tau_{\infty} \\
(\mathrm{ps})\end{array}$ & $\begin{array}{c}W_{A} \\
(\mathrm{~kJ} / \mathrm{mol})\end{array}$ \\
\hline 0 & 5.64 & 57.2 \\
100 & 1.66 & 57.2 \\
200 & 0.53 & 64.1 \\
300 & 0.55 & 64.2 \\
400 & 0.42 & 64.8 \\
\hline
\end{tabular}

Apparently, the relaxation process we have observed is of the $\beta$-type. There are three reasons for this statement:

- the value of $W_{A}$ is the nearest to the values declare for this type of process in literature,

- temperature dependence of the relaxation time does not follow the VFT equation (12) but the Arrhenius law,

- the temperature coefficient of permittivity increment $C_{G} \Delta \varepsilon / \varepsilon_{0}$ (equation 10) is negative [6].

The activation energy $W_{A}$ of the observed process undergoes only a very small change with the absorbed dose. By testing the equality of the values of energy in the third column of Table 1 we found, that this change has no statistical significance. In this test we used the confidence coefficient of 0.95 . It means that the activation energy of the $\beta$-process does not depend on the absorbed dose.

The second important parameter of the process is the permittivity increment $C_{G} \Delta \varepsilon$. It shows a significant decrease with the absorbed dose. A possible explanation of this decrease can be reduction of the number of movable

Table 2. Temperature coefficients of the dielectric parameters at $T_{R E F}=323 \mathrm{~K}$

\begin{tabular}{lcccccccc}
\hline & \multicolumn{2}{c}{$C_{G} \varepsilon_{\infty} / \varepsilon_{0}$} & \multicolumn{2}{c}{$C_{G} \Delta \varepsilon_{\infty} / \varepsilon_{0}$} & \multicolumn{2}{c}{$\alpha$} & \multicolumn{2}{c}{$\beta$} \\
\hline $\begin{array}{l}\text { Absorbed dose } \\
(\mathrm{kGy})\end{array}$ & $p_{\text {REF }}$ & $k_{p}$ & $p_{\text {REF }}$ & $k_{p}$ & $p_{\text {REF }}$ & $k_{p}$ & $p_{\text {REF }}$ & $k_{p}$ \\
\hline 0 & $(\mathrm{ps})$ & $(\mathrm{K})$ & $(\mathrm{ps})$ & $(\mathrm{K})$ & $(-)$ & $10^{3} K$ & $(-)$ & $10^{3} K$ \\
100 & 0.179 & 712 & 0.584 & -15.3 & 0.155 & -6.94 & 0.90 & -2.01 \\
200 & 0.169 & 647 & 0.477 & -179 & 0.140 & -7.64 & 0.83 & -2.31 \\
300 & 0.157 & 586 & 0.378 & -510 & 0.090 & -13.3 & 0.75 & -2.93 \\
400 & 0.147 & 468 & 0.333 & -777 & 0.070 & -17.8 & 0.72 & -3.13 \\
\hline
\end{tabular}


dipoles in a unit volume. It is probably a consequence of new bonds created by radiation (cross linking). Such a behaviour concerning the $\beta$-process in polymers is known from some previous research works [10]. Thermo oxidative degradation induced by radiation is in our case improbable as it decreases the activation energy of the process.

The rest calculated parameters $(\alpha, \beta)$ affect mostly the shape of the loss component of the complex capacitance. They are influenced by the cooperative phenomena and distribution of the relaxation times. We found, that after radiation the relaxation spectrum become narrower in its low frequency part (decrease of $\alpha$ ) and wider in its high frequency part (decrease of $\beta$ ). The physical interpretation of these facts will require a detailed material analysis.

\section{CONCLUSIONS}

A VLF measuring system was realized for diagnostic measurements of cables intended for use in environment of a nuclear power station. The equipment is capable of measuring samples with different capacitances from about $100 \mathrm{pF}$ to several hundred $\mathrm{nF}$. The system uses a commercially available instrument, and a few standard electronic components. This measurement system has been found useful in detecting changes in the irradiated XLPE cable insulation. According to the results of our measurements, dielectric relaxation process of the $\beta$-type is present in the XLPE cable dielectric already in the non-radiated state. Radiation weakens the process in such a way that the number of movable dipoles decreases in consequence of an additional cross-linking of the polymer chains. Any process of the thermo-oxidative degradation after radiation has not been identified in our XLPE cable specimens. As the shape parameters of the $\beta$-process have changed significantly with the absorbed dose, we suppose that the process is influenced by some cooperative phenomena. This can be a subject of the future work. As for the practical use, our results proved that the dielectric measurements in the VLF range could be effectively used for estimation of the absorbed dose in the cross-linked polyethylene cables stressed by radiation.

\section{Acknowledgments}

This work has been supported by Scientific Grant Agency of the Ministry of Education of Slovak Republic and the Slovak Academy of Sciences under project VEGA No. $1 / 0445 / 10$.

\section{REFERENCES}

[1] ZAENGL, W. S.: Dielectric Spectroscopy in Time and Frequency Domain for HV Power Equipment, , 12th Internat. Symposium on High Voltage Engineering - ISH 2001, Bangalore, India, 20-24 August 2001, 1-10.

[2] COLE, K. S.-COLE, R. H.: Dispersion and Absorption in Dielectrics, I. Alternating Current, J. Phys. Chem. No. 9 (1941), 341-351.

[3] HAVRILIAK, S.-NEGAMI, S. : A Complex Plane Analysis of -Dispersions in Some Polymer Systems, J. Polymer Sci. C 14 (1966), 99-117..

[4] JONSCHER, A. K.: The Universal Dielectric Response and its Physical Significance, IEEE Trans. EI. 27 (1992), 407-423.

[5] HELGESON, A.: Analysis of Dielectric Response Measurement Methods and Dielectric Properties of Resin-Rich Insulation During Processing, PhD Thesis, KTH Stockholm, Sweden (2000), $22-24$.

[6] WUBBenhorst, M.-VAN TURnhOUT, J.: Analysis of Complex Dielectric Spectra. I. One-dimensional Derivative Techniques and Three-dimensional Modelling, Journal of Non-Crystalline Solids 305 (2002), 40-49.

[7] SCHÖNHALS, A.: Dielectric Spectroscopy on the Dynamics of Amorphous Polymeric Systems, Novocontrol Application Notes, No.1 (1998), 1-16.

[8] HOLLENBECK, K. J.: A Matlab Function for Numerical Inversion of Laplace Transforms by the De Hoog Algorithm http://www.isva.dtu.dk/ staff/karl/invlap.htm.

[9] SCHÖNHALS, A.-KREMER, F. : Analysis of Dielectric Spectra, Broadband Dielectric Spectroscopy, Springer-Verlag, Berlin (2003), 59-98.

[7] SUlJovrujic, E.-STAMBOLIEV, G.-KOSTOSKI, D. : Dielectric Relaxation Study of Gamma Irradiated Oriented Low-Density Polyethylene, Radiation Physics and Chemistry 66 (2003), 149-154.

Received 22 September 2009

Vladimír Ďurman (Ing, PhD), born in Nové Mesto nad Váhom, Slovakia, in 1946, graduated from the Faculty of Electrical Engineering, Slovak Technical University, Bratislava, at Electrotechnology branch in 1968, and received the CSc (PhD) degree in Electrotechnology at the same university, in 1979. At present he is a research worker at the Department of Electrotechnology, Faculty of Electrical Engineering and Information Technology. He is engaged in research of dielectrics and dielectric properties of materials.

Jaroslav Lelák (Doc, Ing, PhD) was born in Piešt'any in 1951. He graduated from the Slovak University of Technology, Faculty of Electrical Engineering in 1974. He received PhD degree in 1985 in Moscow Institute of Power Engineering. In 1996 he was appointed Associate Professor on Department of Electrotechnology. His main field of interest is degradation processes in insulation systems, durability, and reliability of electrical power systems and prophylactic measurements on high voltage equipment. He is reading lectures from subject Optical and metallic cables, Technology of electrical machines and High voltage technique. 